\title{
BMJ Open Factors influencing career progress for early stage clinician-scientists in emerging Asian academic medical centres: a qualitative study in Singapore
}

\author{
Sungwon Yoon, ${ }^{1,2}$ Woon-Puay Koh, ${ }^{1,2,3}$ Marcus E H Ong, ${ }^{2,4}$ Julian Thumboo ${ }^{2,5}$
}

To cite: Yoon S, Koh W-P, Ong MEH, et al. Factors influencing career progress for early stage clinician-scientists in emerging Asian academic medical centres: a qualitative study in Singapore. BMJ Open 2018;8:e020398. doi:10.1136/ bmjopen-2017-020398

- Prepublication history for this paper is available online. To view these files, please visit the journal online (http://dx.doi. org/10.1136/bmjopen-2017020398).

Received 2 November 2017 Revised 26 December 2017 Accepted 9 January 2018

Check for updates

${ }^{1}$ Center for Clinician-Scientist Development, Duke-NUS Medical School, Singapore, Singapore

${ }^{2}$ Programme in Health Services and Systems Research, Duke-

NUS Medical School, Singapore,

Singapore

${ }^{3}$ Saw Swee Hock School of Public Health, National University of Singapore, Singapore, Singapore

${ }^{4}$ Department of Emergency

Medicine, Singapore General Hospital, Singapore, Singapore ${ }^{5}$ Department of Rheumatology and Immunology, Singapore General Hospital, Singapore, Singapore

Correspondence to Dr Sungwon Yoon; sungwon.yoon@duke-nus. edu.sg

\section{ABSTRACT}

Objectives To explore the factors that influence career progress for early stage clinician-scientists and to identify ways to mitigate these factors in the context of emerging Asian academic medical centres (AMCs).

Design Qualitative interviews and thematic data analysis based on grounded theory.

Setting and participants Five focus group interviews comprising 29 early career clinician-scientists who have received their first national-level career development award in Singapore.

Results Clinical priorities represented an overarching concern with many reporting the difficulty in delineating responsibilities between clinical care and research. Additionally, there was a prevailing perception of the lack of support for research at the institutional level. Participants tended to identify mentors through their own efforts in a relatively haphazard manner, often owing to the dearth of role models and perceived inadequacy of reward systems for mentoring. Support from mentors was thought to be limited in terms of targeted scientific guidance and long-term commitments to the relationship. Most of the participants expressed concerns about how they could secure the next level of funding with diminishing confidence. Notably, the work-life balance was neither conceptualised as a 'barrier' to successful pursuit of research career nor was it translated into the reason for leaving the dual clinical-research career pathway. Conclusions Results revealed specific limitations presented by the research environment in newly emerging Asian AMCs. To retain a vibrant clinician-scientist workforce, additional measures are needed, aiming to improve institutional culture of research, build mentoring networks, adopt effective tools for tracking career progress and provide a clear and viable career progression path for clinician-scientist. Further research might explore the cross-cultural differences in managing work-life balance in academic medicine.

\section{INTRODUCTION}

Early career clinician-scientists in academic medicine are the innovators of the future. They bring fresh ideas and technologies to existing clinical research and pioneer new areas of investigation. ${ }^{12}$ Despite the important

\section{Strengths and limitations of this study}

This is the first national study that comprehensively examined the current experiences and perceptions of clinician-scientists towards pursuing a research career in Asia.

- There is a dearth of studies for early stage clinicianscientists in non-North American academic medical centres.

- The qualitative method allowed for more nuanced accounts of the career transition for early stage clinician-scientists.

- We limited our participants to practicing clinicians with rising research interest for a given career path and therefore our results may not be applicable to individuals with non-clinical background seeking careers in academic medicine.

- We are unable to claim that results from this study based on a single country study are wholly generalisable to other emerging Asian academic medical settings.

role played by aspiring young clinician-scientists in academic medicine, concerns have been raised over the past decades, particularly in North America, about the failure to retain motivated early career clinician-scientists in medical institutions to continue their pursuit in research. Narratives such as 'endangered species' ${ }^{3}$ and 'leaky pipeline" ${ }^{4}$ have invariably portrayed the increasingly challenging conditions placed on the survival of early career clinician-scientists and consequently, the sustainability of academic medicine. ${ }^{5}$

A growing body of research indicates that clinician-scientists encounter a range of significant challenges that hamper their career progress in research. While failure to obtain independent funding contributes significantly to attrition from the career path for early stage investigators, ${ }^{67}$ other issues beyond funding have been also noted. They include pressure on clinicians to increase clinical productivity, inadequate resources 
to protect research time, difficulty covering loans with low salary, unsupportive environment and inadequate accessibility of research infrastructure such as database, biological archives and capability of computing facilities. Lack of mentorship and role models, as well as personal factors, are thought to have contributed to the barriers to successful transition of new investigators to independence. ${ }^{8-14}$

While the existing literature provided important knowledge about the barriers faced by clinician-scientists, studies often included a whole spectrum of clinician-scientists from early to advanced careers, rather than focusing on more homogenous groups in terms of career progress. Additionally, the existing literature is primarily characterised by a large volume of non-empirical publications (eg, opinion pieces, letters and commentaries) based on anecdotal cases and not well substantiated by evidence that has been collected systematically. Furthermore, studies that have used a quantitative method tend to focus on career satisfaction and outcomes of career development programmes, ${ }^{9} 1516$ whereas qualitative studies typically provide descriptions of one or more specific components of career progression, such as mentoring, work-life balance and resilience. ${ }^{17-22}$ One study conducted by Robinson and colleagues provided a comprehensive range of factors that facilitated career success for career development awardees in the United States. ${ }^{8}$ However, there is a dearth of studies for early stage clinician-scientists in non-North American academic medical centres (AMCs). In particular, no research so far has been undertaken on the experience of early career clinician-scientists in the context of newly emerging Asian academic medical settings.

We aimed to fill this gap by generating an in-depth understanding of the career transition for early stage clinician-scientists in emerging Asian AMCs. Specifically, this study sought to explore the nature of experience and barriers to career progression perceived by clinician-scientists who have received the first national-level career development award in Singapore. An understanding of the limitations presented by the current research environment in academic medicine will provide an insight into how best to overcome these barriers. Importantly, this study may inform future efforts to establish a stable pipeline that nurtures clinician-scientists in other newly emerging AMCs.

For this study, clinician-scientists are defined as clinicians practicing in Singapore, who have received the first national-level career development award that provides both salary and research funding to do research in their respective institutions.

\section{METHODS}

\section{Setting: career development award in Singapore}

In Singapore, despite the government's emphasis on biomedical sector as a key engine of economic growth, the number of clinician-scientists has generally remained low. ${ }^{23}$ In order to build a cadre of clinician-scientists, the National Medical Research Council (NMRC), the primary funder of biomedical research and training in Singapore, initiated a competitive research career award, named the Transition Award (T-award), in 2012. This mentored award programme aims to assist the career development of early stage clinician-scientists by providing 3 years of salary support and a research grant for a specific project. The T-award stipulates that applicants must have received at least 2 years of intensive research experience in relevant local or overseas universities, research institutes and centres. Further, they must be at the early stage of their research careers in not having received any substantial competitive research funding defined by a specific quantum. The T-award is given primarily to an individual who holds a clinical qualification (MBBS, MD, MDS or equivalent) and registered in Singapore Medical Council's register of specialists. However, the following two categories of applicants are considered as exception on a case-by-case basis: (1) non-medically trained and $\mathrm{PhD}$ holders working in human clinical research (eg, PhDs in areas of biostatistics, epidemiology, behavioural science, nursing, pharmacy, psychology and allied health); (2) individuals who demonstrate completion of specialist training in foreign countries and are not registered and working as specialists in Singapore. ${ }^{24}$ Between 2012 and 2016, the T-award programme produced 46 awardees from different clinical specialty areas, and 10 individuals received the T-award as 'exception cases'. In parallel, the development of two AMCs in Singapore has further provided clinician-scientists with an environment conducive to pursing excellence in clinical academic medicine. ${ }^{25-27}$

\section{Data collection}

From the list of 46 recipients awarded between 2012 and 2016, we excluded 10 awardees who received the T-award as 'exception cases' and one who had not commenced work on his T-award at the time of the study. We excluded these individuals for the following reasons: first, 'exception cases' who are non-medical scientists doing clinical research do not face multiple competing demands at work that the clinician-scientists face as the latter have a career combining clinical work with concurrent research. Therefore, the perspectives of these 'exception cases', including the factors influencing their career progression, may be different from those of the clinician-scientists. Second, recipients as 'exception cases' are generally better trained than their clinician-scientist counterparts in research, as more from this group have MSc or $\mathrm{PhD}$ in research compared with the clinician-scientists. Finally, we excluded the awardee who had not commenced work on his award as he would not have experienced the challenges encountered by early stage clinician-scientists in working on their T-award research projects.

We purposively approached 35 eligible T-award recipients via email and 29 individuals took part in one of the five focus groups, each involving three to seven 


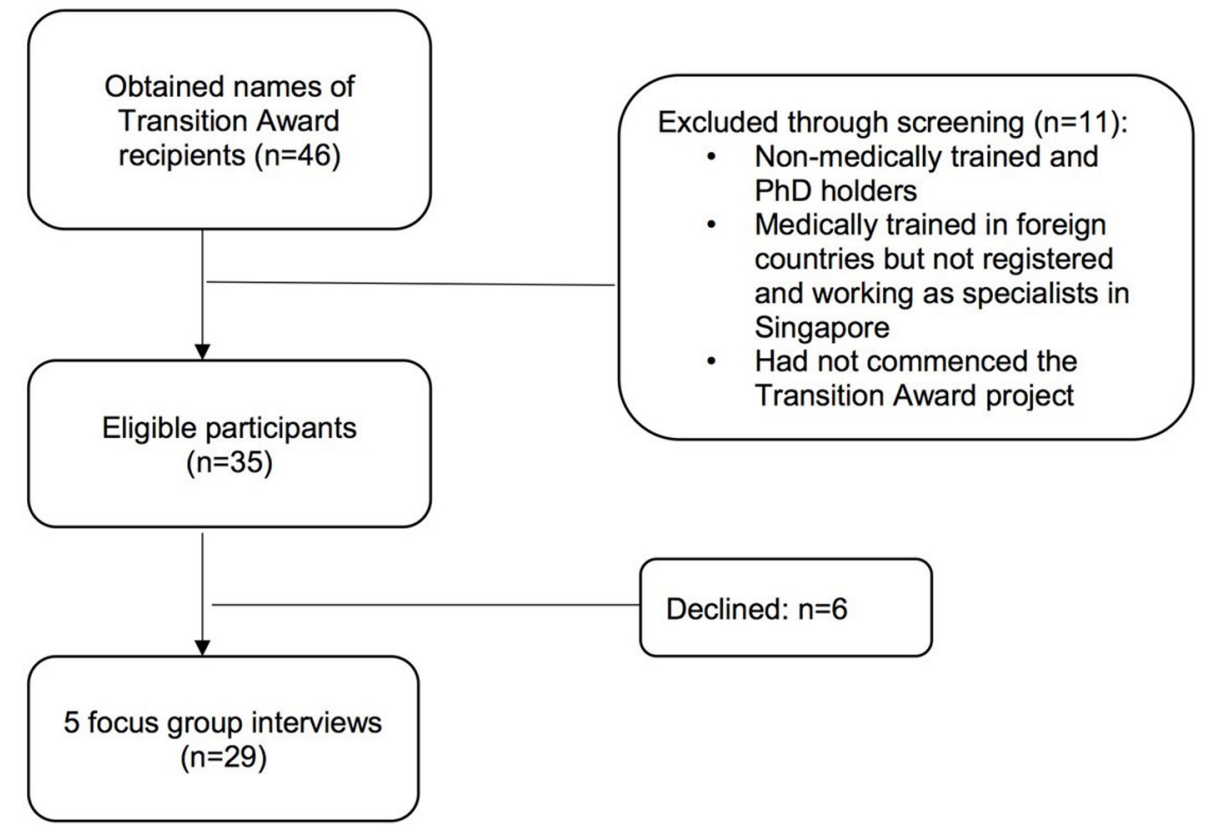

Figure 1 Flowchart of study process

participants. Reasons for refusal by six eligible recipients included not being available or interested (figure 1). In order to ensure a degree of commonality and shared experience among participants in each group, the focus groups consisted of participants from the same host institution. We developed an interview guide with openended questions to solicit the participants' experience and perceptions of the clinician-scientist career path. Between June and July 2016, the first author, who has extensive experience in qualitative research, conducted focus group interviews in a conference room. In Singapore, English is the first language and hence all interviews were conducted in English. Each focus group lasted approximately 75-90 min and were audio-recorded. We differentiated speakers by giving each participant a numeric identifier in the focus groups. We achieved data saturation ${ }^{28}$ after four focus group interviews. All participants gave informed consent.

\section{Data analysis}

We transcribed the interviews verbatim and analysed the transcripts thematically based on a grounded theory approach. ${ }^{29}$ In the first step, the first author (SY) and her assistant, who was a trained qualitative researcher, independently identified themes using a constant comparative method in a subset of transcripts and developed codes from these themes. This process allowed themes and explanations to arise inductively from the data. In the second step, all authors (SY, WPK, MEHO and JT) had consecutive rounds of iterative discussion, through which any discrepancies in interpretation were reconsidered in order to reach an agreement. This allowed for intercoder clarification of themes and coding, thus enhancing validity and reliability. We compiled a further classification, in which several codes and categories were refined and recoded. Alternative interpretations were also incorporated into the analysis. In the third step, the first author (SY) drew up a summary of themes with verbatim quotes aimed at exemplifying the codes and categories. Using the QSR NVivo 11 software, we retrieved quotes associated with each theme to determine the difference by participants' characteristics (eg, gender) and reliability of categories. To ensure the rigour of the study, we anchored our methodology according to the Consolidated Criteria for Reporting Qualitative Research (COREQ) checklist. ${ }^{30}$

\section{RESULTS}

Among 29 participants, 18 (62\%) were men and 17 $(58 \%)$ were at the rank of a consultant or the equivalent when they received the T-award. The mean age at the time of award was 39 years, with $28 \%$ at ages over 40 years. Approximately half of the participants $(14 / 29,48 \%)$ had obtained research training fellowship from the NMRC to pursue training in research prior to receiving the T-award (table 1).

We identified several recurring and prominent themes with regard to the factors influencing career development perceived by early stage clinician-scientists. A key theme that emerged was that despite being positive about embarking on a research career, many participants found it challenging to pursue the research career path they wanted. Factors influencing research career development essentially related to the research role and support in multiple realms.

\section{Coping with clinical priorities}

On the whole, although participants recognised that having protected time for research was pivotal to career success, many experienced difficulty maintaining research activity. As shown in table 1, nearly two-thirds of the participants dedicated less than $60 \%$ of their time 
Table 1 Characteristics of focus group participants $(n=29)$

\begin{tabular}{lc}
\hline Characteristic & $\mathbf{N}(\%)$ \\
\hline Gender & $18(62.1)$ \\
\hline Male & $11(37.9)$ \\
\hline Female & $1(3.5)$ \\
\hline Clinical appointment when received T-award \\
\hline Registrar & $5(17.2)$ \\
\hline Associate Consultant & $17(58.6)$ \\
\hline Consultant & $5(17.2)$ \\
\hline Senior Consultant & $1(3.5)$ \\
\hline Clinician-Scientist & \\
\hline Age when received T-award & $3(10.3)$ \\
\hline $31-35$ & $18(62.1)$ \\
\hline $36-40$ & $5(17.2)$ \\
\hline $41-45$ & $2(6.9)$ \\
\hline $46-50$ & $1(3.5)$ \\
\hline $51-55$ & $14(48.3)$ \\
\hline Acquisition of training fellowship prior to T-award \\
\hline Yes & $15(51.7)$ \\
\hline No & \\
\hline Intention to pursue a research career & $22(75.9)$ \\
\hline Yes & $7(24.1)$ \\
\hline No or uncertain & $1(3.4)$ \\
\hline Actual time dedicated to research activity & \\
\hline $0 \%-20 \%$ & $11(37.9)$ \\
\hline $21 \%-40 \%$ & $1(34.4)$ \\
\hline $41 \%-60 \%$ & \\
\hline $61 \%-80 \%$ & \\
\hline $81 \%-100 \%$ & \\
\hline
\end{tabular}

to research. A myriad of conflicting demands appeared to have impeded balancing clinical duties and research activities. When asked about conflicting demands, many pointed out the perceived pressure to perform clinical duties and frequent intrusions owing to the overwhelming volume of patient encounters and on-call duties. Other competing demands include being assigned to administrative leadership roles in the department, teaching obligations and concerns about losing clinical competency, particularly for those working in surgical specialties. The following quotes illustrate that many things eroded into the protected time:

Even though on paper I'm only meant to be doing $30 \%$ [of clinical work], on the ground within my department, there is a requirement or a need because essentially no one backfills, no one comes in to take over your job. So there's still work that needs to be done (Participant 13).

Because I am doing research, I was appointed as the research director of my department. Half of my time is spent on sorting out the problems that are inherent in the department and the institution (Participant 2).

Therefore, longer work hours were unavoidable in order to meet the demands and expectations of both clinical and research work. By and large, there was a common perception that research was not adequately valued by the medical institutions, and the insufficient protected time was attributable to the organisational emphasis on clinical work over research activities.

The main issue is that clinical work is always number one in the current institution I'm at. So research is always look upon as a life of leisure or something extra. At the end of the day, if you have to drop something, research always gets dropped first. (Participant 22)

The low institutional support for research also hampered the manpower backfill process. Although funding was made available to cover T-awardees' research time, clinical replacement rarely took place, at times resulting in conflict with and resentment from other clinicians. Consequently, participants often found themselves in a situation where peer clinical colleagues viewed their time spent on research as 'time off'. As one participant noted:

For example, my colleague said 'Oh okay, we need to cut down your clinic now in order to give you protected time? So I have to ask somebody else to take over your clinic?' Begrudgingly then, that person does it, and then now people are not happy with me. (Participant 1)

The clinical priorities also appeared to have a considerable ramification for career advancement. Participants commonly felt that current metrics for promotion was unfair to clinician-scientists and the reason for some to eventually give up research. The expectation to do well both as a clinician and a scientist under the existing promotion metrics generated an overall sense of frustration in pursuing a research career. As the following participants stated:

It startled me to realize fairly recently that to move on and become a senior consultant, I may be held back. It impedes career transition because I'm not compared to other clinician scientists. I'm compared to other straight scientists or straight clinicians. Actually, I'm compared to both, and I can never compete with either of those. That's a big challenge. (Participant 8)

It is a de facto agreement right now that if you are an almost full time research clinician, you can't possibly be promoted equally on the same track. That already puts you at a big disadvantage. There seems to be no hurry to try and crack that, which is going to be detrimental in the long run because I can see that my junior colleagues have a lot of reluctance to take up academic careers. (Participant 12) 
Fighting the battle on your own: need for streamlined administrative support

Across focus groups, participants consistently identified adequate administrative and logistical support for research as key to research productivity and career progress. Regrettably, many faced a lack of administrative support particularly in the initial period of starting their projects. Some participants faced little guidance on where and how to seek help regarding research-related tasks, such as grant management and human resources matters. Others perceived the support system and protocols to be highly complicated and inflexible, resulting in unnecessary waiting time and paperwork. As the following quotes demonstrate, having to navigate the research-related administrative tasks alone was an additional source of stress for many participants:

There's a lot of red tape. There're so many things to apply for. If you want to do animal studies and you have an external collaborator, you have to get a research agreement or complete tons of paperwork. At every point, everybody is saying oh you should do this, you need to talk to somebody else. You really feel like you're fighting the battle on your own. You're winning this grant and bringing sort of recognition to the institution, and yet you're treated like a liability rather than an asset. (Participant 19)

You have to sign so many legal and financial documents with the [host] institution before you can even start something, and they are not straightforward. Recently, my institution has revamped the financial billing system to streamline the work for administrators but it creates double work for researchers....it is a nightmare...every time you ask people for help, there will be another four requirements to fulfil before you can get the help. (Participant 24)

\section{Opening the door: seeking out mentors and collaborators}

Participants identified mentorship as a catalyst for their research career progress. When asked when and how mentorship plays a critical role, the nature of the perceived benefits from effective mentorship appeared to be different according to participants' personal needs and circumstances. For example, some participants appreciated the provision of vital resources such as laboratory space while others indicated that mentors were useful in protecting them from challenging situations:

My mentor is a resource mentor. I was very lucky to find him as my mentor as he provided lab space, samples and all other consumables. That facilitated the progress of my research. To me, this type of mentorship is very vital. (Participant 5)

When I started my T-award, I got my mentor who encouraged me to do research. He is the head of my department. Because your boss is directly involved in the [performance] assessment, this has made a great difference. Beyond encouraging research, when the department questioned why Doctor X had not been doing the [clinical] work, he was able to support me. (Participant 18)

Participants commonly mentioned that multiple types of mentors were required to serve various needs that ranged from knowledge-specific guidance to career advice.

The mentor serves a few purposes, and you may need different mentors beyond the primary mentor for that grant, because different supervisors and mentors have different strengths and skill sets. (Participant 11)

Many participants actively looked for international mentors. By and large, while scientific guidance from international mentors was seen helpful, some participants felt concerned about the potential for competition with their international mentors in the long run.

If you are building cohort and there is nobody guiding you on how to look at the disease in particular, that is the problem. With the mentor overseas, they can still give you some of the advice, but some of the time, you are the competitor. You have to be careful about which overseas mentors you will be approaching because they will end up being your competitors. (Participant 28)

Recurring annotations from the participants were that locally, there were not enough mentors who could guide them in their content-specific research projects. While many saw the absence of local expertise as a crucial concern, a small group of participants asserted that the underlying reason for insufficient mentors was the absence of mentor-matching system for junior researchers to identify suitable mentors. Additionally, it was generally perceived that there was inadequate academic reward to incentivise mentors to devote the time to develop effective mentorship relationships.

I had applied for T-award three times before I got it. So people asked me why I failed so many times. In my clinical specialty, nobody does research. So the difficulty is you can't find mentors in the same specialty. It's impossible because no one else has gone through the same path. So it's totally unchartered. (Participant 17)

There's the kind of culture that taking on someone as a mentee is extra work for which they don't get recognized and there's no value added for them to bother. Or people in my discipline don't have that kind of belief that they need to nurture the next generation of clinician-researchers...The only time I met my mentors is when I applied for my T-award. Since I received the T-award, I have been moving on without mentors. (Participant 6)

There was also a lack of network of collaborators and research resources for the T-award recipients, with 
resultant negative impact on research productivity. For example, having to work alone restricted access to an integrated array of manpower, research facilities, databases and even a dedicated office space for research. As one participant described:

I lost one research staff. He is a research pathologist with a unique set of skills that I cannot easily find, and I waited for half a year for another candidate to appear. That's lost time. If your research group is small, or if you don't have collaborators, there's no support. (Participant 20)

Some participants attempted to develop research networks locally and internationally by proactively investing time to engage with people. At the same time, a few of participants contemplated their experience in sourcing for collaborators.

It is no secret that many of us are literally just standing on our own firefighting ... I realized that I just have to go out, beg, borrow and make friends with people from all different institutions. That's the only way I can survive. I did have so much struggle in networking. (Participant 9)

We have very good clinical skills but we're lacking solid scientific support and collaboration. There are some [lab] facilities out there, but it's like working with a collaborator. Then it's adding to a lot of uncertainty in the string of collaboration. You don't know if the collaborators are trustworthy or they will give you high priority to do your project or not. (Participant 13)

\section{Managing transition: sense of insecurity in future career path}

One important theme in this study was the prevailing perceptions of career uncertainty. While many had motivation and aptitude for research, they were often discouraged by the lack of clarity on routes to further their research career. Participants noted that planning a research career path was straightforward in the early stage when they embarked on applying for the T-award. However, due to low success rate in the acquisition of independent funding after the T-award, participants began to lose confidence in their ability to secure further research funding. The apparent lack of confidence was illustrated by the following participants.

It's great that someone getting a T-award can be placed on a clinician-scientist path. But if your T-award lapses and you don't get the [next level of] Clinician-Scientist Award, does that mean that you come off the career path and go back again to full time clinical work? Then once you go back to full time clinical work, not only your research thinking will suffer tremendously but you also won't be able to fund your own research team. (Participant 3)

After your research stops, they may throw you back to do all the rest of the clinical work, which you have been out of touch for the last five to ten years, and you will struggle. The more you give up, the more difficult it will be for you to come back later on. (Participant 1)

Participants consistently maintained that 3-year funding duration of the T-award was insufficient to get the research project up and running, analyse and write manuscripts accepted in high impact journals and plan a strategic research agenda for further research funding application. This created a sense of insecurity in their future career paths. Indeed, approximately one in four participants (24\%) expressed uncertainty about pursuing a research career (table 1 ).

As a new junior investigator, you need to set up a new lab. But you have a lot of pressure to publish high impact factor papers at the end of the term that is obviously less than threeyears...It is something that is difficult to meet for a lot of people because it takes time to get their research off the ground. It took me close to twoyears to actually get the animals in, get the experiments going, and get the data generated. (Participant 20)

I got my T-award at the end of last year. I feel like once you got it, the clock is ticking. Then you haven't started but you already feel it's like a tightened schedule to finish and to gain enough record to build up for the next step. (Participant 24)

\section{Having the right skills and mindset to achieve work-life balance}

Participants commonly acknowledged the importance of family support in research career success. In particular, they viewed family support as being instrumental to managing the substantial demands from both clinical and research workloads.

A critical component is support from family because if I work till late at night having to stay back at the hospital, someone has to look after my son. Without support from my family I wouldn't have reached this far actually. There's just simply no way, no matter how family-oriented the institution can claim to be. (Participant 15)

Notably, competing expectations of career and personal/family life were not viewed as something to lament, let alone conceptualised as a factor impeding one's research productivity and career progression. Rather, it was a representation of how one approached the challenges associated with work-life issues. As the following quotes illustrate, many participants made sense of the balance between personal commitments and work as a matter of developing resilience and skill sets and displayed a personal coping strategy to negotiate the reality.

If you didn't have the basic skill sets of managing your family and your stuff, I don't think you're going to get 
to where you're at now. From my experience, worklife balance is you just sleep less and do you work when your family is sleeping. That's work-life balance. I think your time management is important. Also, you need to be very focused and not get distracted. (Participant 4)

I figured you can manage the work-life balance if you have the sheer mindedness to just keep going on. Then, that will get you pass hurdles and find the way to get all things done... I think it [managing worklife balance] is very much down to you. That's why some people who are very talented actually leave [clinician-scientist career path] while those who may not be so talented but have a lot more grit and determination eventually succeed. (Participant 29)

This indicates that certain sacrifices in personal/family life are necessary to become successful clinician-scientists. While few female participants, when elicited, expressed the challenges of having to respond to the demands of both work and family commitments, no marked difference in the attitude towards work-life balance was observed between men and women in this study.

\section{DISCUSSION}

This study provided unique descriptions of the ways in which early career clinician-scientists experienced and managed the career transition in the context of emerging Asian AMCs. To our knowledge, this is the first national study to comprehensively examine the current experiences and perceptions of clinician-scientists towards pursuing a research career in Asia. In addition to the observations found in previous studies, our findings revealed important insights into the specific limitations presented by the current academic and research environment in Asia.

Our findings resonated with those reported by other researchers that clinical priorities represented an overarching concern and the main cause for a diminishing motivation for pursuing a research career. ${ }^{6} 81112$ 31-34 Many participants reported difficulty delineating responsibilities related to clinical care and research, resulting in insufficient protected time to be successful in research endeavours. Research suggests that protected time for research is critical to career satisfaction and clinician's research productivity. ${ }^{832} 33$ This indicated a need for support for clinician-scientists to secure dedicated research time. At the same time, what emerged salient from the account of participants was that there appeared to be a widespread climate among the local institutions in which research pursuit was little valued. This was manifested in a range of areas: being unsympathetic to the need for research and role of clinician-scientists at the institutional level; slower promotion than clinical peers in the same specialty with reduced remuneration and poor administrative and logistical support for research-related activities. The fundamental issue therefore is not merely a matter of securing protected time for research in our setting. Rather, it is the cultural and institutional elements inherent in the existing system that prioritises clinical work over research and that consequently serves to constrain those committed to a research career path. The interplay between institutional inertia and drivers for change in academic medicine underlines that tackling institutional culture will require systemic actions that are further reaching and more difficult than advising early career clinician-scientists on how to navigate institutional challenges. Although a large-scale change may be daunting, an overarching strategy involving key stakeholders to increase the perceived value of clinician-scientists should be implemented.

Our study found that despite the recognised importance of mentorship in career success and satisfaction in academic medicine, ${ }^{6-10} 143235$ few participants received appropriate mentoring across the trajectory of the T-award. Participants perceived diverse mentoring needs, but recognised the difficulty in having a mentor with different skill sets and functions. International mentors were often sought by some T-award recipients to address unique needs when receiving inadequate support from mentors from within their own institutions. Notably, our participants tended to identify mentors through their own efforts in a relatively haphazard manner rather than seek a more personalised and comprehensive mentorship. A variety of factors may have contributed to this but a dearth of role models and inadequate reward systems for mentoring may have resulted in the aforementioned haphazard manner of selecting mentors. Consequently, the value of using mentors for help was primarily driven by interim and temporary considerations such as having a mentor endorse the T-award application, securing protected time for research from clinical duties and using mentor's laboratory space. It became apparent that even for those who managed ongoing interaction with mentors, support from mentors was found to be limited in terms of targeted scientific guidance and long-term commitments to the relationship. This is in contrast to the situation in established AMCs in North America, ${ }^{17} 1921$ where there is a shared value and alignment of research projects between mentor and mentee and hence mentorship does evolve and grow as the mentees move towards independence. Our findings suggest a growing need for a structured mentoring model to systematically support mentees' individual needs and preferences as well as to publicly recognise mentoring efforts. Given a relatively small pool of senior mentors readily available in our setting, this model may further benefit from what other researchers have described as the ' $360^{\circ}$ mentoring network', ${ }^{18}$ which is a network that represents different types of mentors based on competencies with varying career stages and status levels.

Another key finding of the current study is that we did not observe prevailing perceptions of work-life incompatibility in academic medicine, which is in stark contrast to the prior studies conducted elsewhere. ${ }^{8122236}$ While 
participants often acknowledged the importance of family support for their careers, the work-life balance by itself was neither generally conceptualised as a 'barrier' to the successful pursuit of a research career nor was it translated into the reasons for leaving the dual clinical-research career pathway. Also, there was no distinct difference in the perception of work-life balance by gender. What is noteworthy and potentially intriguing is that many saw certain individual qualities such as tenacity, hard work and appropriate skill sets as something that could overcome challenges associated with personal-professional commitments. Research suggests that contrary to Western culture in which the self-value is relatively emphasised, Asian culture is largely influenced by collectivism that emphasises developing and maintaining harmony within family and group. ${ }^{37}$ As a result, in Asian culture, information is communicated in a polite, indirect and restrained manner so as to protect personal relationship from public embarrassment. It could be the case that our participants were sensitive to issues associated with family and personal circumstances and hence felt reticent to express their views in an explicit and direct manner. ${ }^{38}$ It is also possible that the primary value of perseverance in achieving one's goal in Asian culture may have influenced how our participants approached the issue of work-life balance. ${ }^{37}$ The ways in which early career clinician-scientists perceived and dealt with work-life balance in Asian culture may provide further avenues for examining cross-cultural variations in the management of personal-professional commitments in academic medicine.

Our study also provides strong evidence to support previous research that early career clinician-scientists experience mounting pressures to secure the next level of funding with diminishing confidence and time commitments. ${ }^{6} 8940$ The funding duration of the T-award (ie, 3years) was thought to be insufficient to finish the research project and amass needed publications to be competitive for independent funding. Compared with the counterparts in established AMCs in North America, ${ }^{8-10} 1721$ maintaining continued grant funding was further challenged for our participants by the absence of supportive mentoring relationship and institutional resources that were pivotal in enabling them to remain dedicated to research in the event of career adversity or gap years by providing continued access to shared staff, supplies and other resources. The perceived difficulty in securing independent funding and the possibility of having to 'drop out' of research career placed tremendous pressure on early career clinician-scientists and led to a sense of uncertainty and vulnerability in the academic medicine career path. ${ }^{41}{ }^{42}$ Our findings suggest the need for sustained funding ${ }^{7}$ to enable promising investigators to continue to pursue their productive research endeavours. However, simply providing more funding may be neither sufficient nor feasible. To provide a clearly defined and viable career path for clinician-scientists, AMCs have to adopt effective tools to track their career development and innovate solutions to address the enduring cacophony between clinical duties and research interests.

Findings from this study should be considered in light of several limitations. While we recruited a diverse range of participants in terms of gender, age and clinical rank in order to garner different opinions concerning the early career development, it was not possible to explore in depth the views of certain groups. For example, we did not provide a gender-specific account of career challenges although no distinct gender difference ${ }^{43}$ was observed. Additionally, while the focus group enabled us to observe social interactions and group dynamics, individuals in the group might have influenced comments and thus some participants might have felt intimidated in sharing personal experiences. We limited our participants to practicing clinicians with developing research interest for a dual-career path and therefore our results may not be applicable to individuals with non-clinical background seeking careers in academic medicine or clinically trained scientists who only do research. We are unable to claim that the findings of this investigation based on a single country study are wholly generalisable to other emerging Asian academic medical settings. Despite these limitations, we hope that this study may provide useful information for other settings that embark on fostering a clinician-scientist workforce.

In conclusion, this study sheds light on the challenges and opportunities that early stage clinician-scientists faced in the context of emerging Asian AMCs. Our study is timely in the current environment of efforts to develop a vibrant clinician-scientist workforce in Asia with an aim to build a robust and globally competitive biomedical research infrastructure. ${ }^{44}$ To retain this nascent clinician-scientist workforce, measures required include improving institutional culture of research, building mentoring networks, adopting effective tools for tracking career progress and providing a clear and viable career progression path for clinician-scientist. More broadly, it would be imperative to enhance societal awareness that supporting the success of clinician-scientists is not simply an investment in biomedical advances, but in the future of the nation's health and healthcare delivery as well.

Acknowledgements The authors would like to thank the support from Singapore Health Services (Professor Wong Tien Yin), National University Health System (Associate Professor Allen Yeoh) and National Healthcare Group (Professor Lim Tock Han) for the development of the study. The authors are also grateful to the Transition Award recipients who took the time to participate in the study and to Meixuan Chen and Fion Farn for excellent administrative support.

Contributors SY and WPK conceived the idea and designed the study. MEHO and JT helped specify the research questions. SY did the interviews. SY undertook the data analysis with assistance from WPK, MEHO and JT. SY drafted the initial manuscript. WPK, MEHO and JT critically reviewed the manuscript. All authors read and approved the final manuscript.

Funding This work was supported by the National Medical Research Council Singapore (NMRC/RIE 2020).

Competing interests None declared.

Patient consent Obtained. 
Ethics approval National University of Singapore Institutional Review Board (reference B-16-092).

Provenance and peer review Not commissioned; externally peer reviewed. Data sharing statement № additional data are available.

Open Access This is an Open Access article distributed in accordance with the Creative Commons Attribution Non Commercial (CC BY-NC 4.0) license, which permits others to distribute, remix, adapt, build upon this work non-commercially, and license their derivative works on different terms, provided the original work is properly cited and the use is non-commercial. See: http://creativecommons.org/ licenses/by-nc/4.0/

(C) Article author(s) (or their employer(s) unless otherwise stated in the text of the article) 2018. All rights reserved. No commercial use is permitted unless otherwise expressly granted.

\section{REFERENCES}

1. National Academy of Science. Bridges to independence. Washington, DC: The National Academies Press, 2005.

2. National Academy of Science. Addressing the nation's changing needs for biomedical and behavioral scientists. Washington, DC: The National Academies Press, 2000.

3. Wyngaarden JB. The clinical investigator as an endangered species. N Engl J Med 1979;301:1254-9.

4. Ley TJ, Rosenberg LE. The physician-scientist career pipeline in 2005: build it, and they will come. JAMA 2005;294:1343-51.

5. Schafer Al. The vanishing physician-scientist? Trans/ Res 2010;155:1-2.

6. Tong CW, Ahmad T, Brittain EL, et al. Challenges facing early career academic cardiologists. J Am Coll Cardiol 2014;63:2199-208.

7. Lander B, Hanley GE, Atkinson-Grosjean J. Clinician-scientists in Canada: barriers to career entry and progress. PLoS One 2010;5:5.

8. Robinson GF, Schwartz LS, DiMeglio LA, et al. Understanding career success and its contributing factors for clinical and translational investigators. Acad Med 2016;91:570-82.

9. Goldhamer ME, Cohen AP, Bates DW, et al. Protecting an endangered species: training physicians to conduct clinical research. Acad Med 2009;84:439-45.

10. Bruce ML, Bartels SJ, Lyness JM, et al. Promoting the transition to independent scientist: a national career development program. Academic Medicine 2011;86:1179-84.

11. Kubiak NT, Guidot DM, Trimm RF, et al. Recruitment and retention in academic medicine - what junior faculty and trainees want department chairs to know. Am J Med Sci 2012;344:24-7.

12. Rosier RN. Institutional barriers to the orthopaedic clinician-scientist. Clin Orthop Relat Res 2006;449:159-64.

13. Harrington RA, Califf RM, Hodgson PK, et al. Careers for clinician investigators. Circulation 2009;119:2945-50.

14. Ranieri V, Barratt $\mathrm{H}$, Fulop $\mathrm{N}$, et al. Factors that influence caree progression among postdoctoral clinical academics: a scoping review of the literature. BMJ Open 2016;6:e013523.

15. Sakushima K, Mishina H, Fukuhara S, et al. Mentoring the next generation of physician-scientists in Japan: a cross-sectional survey of mentees in six academic medical centers. BMC Med Educ 2015;15:54.

16. Biondi-Zoccai G, Cerrato E, Peruzzi M, et al. An international survey on taking up a career in cardiovascular research: opportunities and biases toward would-be physician-scientists. PLoS One 2015;10:e0131900.

17. Straus SE, Chatur F, Taylor M. Issues in the mentor-mentee relationship in academic medicine: a qualitative study. Acad Med 2009;84:135-9.

18. DeCastro R, Sambuco D, Ubel PA, et al. Mentor networks in academic medicine: moving beyond a dyadic conception of mentoring for junior faculty researchers. Acad Med 2013;88:488-96.

19. Straus SE, Johnson MO, Marquez C, et al. Characteristics of successful and failed mentoring relationships: a qualitative study across two academic health centers. Acad Med 2013;88:82-9.
20. DeCastro R, Griffith KA, Ubel PA, et al. Mentoring and the career satisfaction of male and female academic medical faculty. Acad Med 2014;89:301-11.

21. DeCastro R, Sambuco D, Ubel PA, et al. Batting 300 is good: perspectives of faculty researchers and their mentors on rejection, resilience, and persistence in academic medical careers. Acad Med 2013;88:497-504.

22. Strong EA, De Castro R, Sambuco D, et al. Work-life balance in academic medicine: narratives of physician-researchers and their mentors. J Gen Intern Med 2013;28:1596-603.

23. Williams RS, Casey PJ, Kamei RK, et al. A global partnership in medical education between duke university and the national university of singapore. Acad Med 2008;83:122-7.

24. National Medical Research Council. Transition award. http://www. nmrc.gov.sg/content/nmrc_internet/home/grant-navigation/talentdevelopment/transition-award.html (accessed 12 Dec 2017).

25. Krishnan RR, $\mathrm{Ng}$ I. Academic medicine: vision to reality. Ann Acad Med Singapore 2013;42:2-4.

26. Soo KC. Singapore's proposed graduate medical school-an expensive medical tutorial college or an opportunity for transforming Singapore medicine? Ann Acad Med Singapore 2005;34:1-3.

27. Tai ES. Clinician scientists in public sector hospitals - why and how? Ann Acad Med Singapore 2013;43:1-3.

28. Creswell JW. Qualitative inquiry and research design: choosing among five traditions. Sage: Thousand Oaks, 1988.

29. Glaser BG, Strauss AL. The discovery of grounded theory: strategies for qualitative research. Chicago: Aldine, 1967.

30. Tong A, Sainsbury P, Craig J. Consolidated criteria for reporting qualitative research (COREQ): a 32-item checklist for interviews and focus groups. Int J Qual Health Care 2007;19:349-57.

31. Milewicz DM, Lorenz RG, Dermody TS, et al. Rescuing the physicianscientist workforce: the time for action is now. J Clin Invest 2015;125:3742-7.

32. Yin HL, Gabrilove J, Jackson R, et al. Sustaining the clinical and translational research workforce: training and empowering the next generation of investigators. Acad Med 2015;90:861-5.

33. Bossé D, Milger K, Morty RE. Clinician-scientist trainee: a German perspective. Clin Invest Med 2011;34:324-9.

34. Clough S, Fenton J, Harris-Joseph $\mathrm{H}$, et al. What impact has the NIHR Academic Clinical Fellowship (ACF) scheme had on clinical academic careers in England over the last 10 years? A retrospective study. BMJ Open 2017;7:e015722.

35. Lingard L, Zhang $P$, Strong $M$, et al. Strategies for supporting physician-scientists in faculty roles: a narrative review with key informant consultations. Acad Med 2017:92:1421-8.

36. Levinson W, Tolle SW, Lewis $\mathrm{C}$. Women in academic medicine. Combining career and family. N Engl J Med 1989;321:1511-7.

37. Hall ET. Beyond culture. New York: Doubleday, 1976.

38. Rubin $\mathrm{KH}$. Social and emotional development from a cultural perspective. Dev Psychol 1998;34:611-5.

39. Bruce ML, Bartels SJ, Lyness JM, et al. Outcome of national caree development program that promotes the transition to independent scientist. Acad Med 2011;86:1179-84.

40. Rubio DM, Primack BA, Switzer GE, et al. A comprehensive careersuccess model for physician-scientists. Acad Med 2011;86:1571-6.

41. Lopes J, Ranieri V, Lambert T, et al. The clinical academic workforce of the future: a cross-sectional study of factors influencing career decision-making among clinical $\mathrm{PhD}$ students at two research-intensive UK universities. BMJ Open 2017;7:e016823.

42. Roberts SF, Fischhoff MA, Sakowski SA, et al. Perspective: transforming science into medicine: how clinician-scientists can build bridges across research's "valley of death". Acad Med 2012;87:266-70.

43. Jagsi R, Griffith KA, Jones RD, et al. Factors associated with success of clinician-researchers receiving career development awards from the national institutes of health: a longitudinal cohort study. Acad Med 2017;92:1429-39.

44. Razak MA. First college for clinician scientists in Asia launched in Singapore: The Straits Times, 2016. 